\title{
Structural Studies on Nonequilibrium Microstructures of Dioctyl Sodium Dodecyl Sulfosuccinate (Aerosol-OT) in p-Toluenesulfonic Acid and Phosphatidylcholine
}

\author{
M. K. Temgire, ${ }^{1}$ C. Manohar, ${ }^{1}$ Jayesh Bellare, ${ }^{1}$ and S. S. Joshi ${ }^{2}$ \\ ${ }^{1}$ Department of Chemical Engineering, Indian Institute of Technology, Bombay, Mumbai 400076, India \\ 2 Department of Chemistry, University of Pune, Pune 411 007, India \\ Correspondence should be addressed to S. S. Joshi, ssjoshi@chem.unipune.ac.in
}

Received 20 April 2012; Revised 8 November 2012; Accepted 12 November 2012

Academic Editor: Jan Skov Pedersen

Copyright () 2012 M. K. Temgire et al. This is an open access article distributed under the Creative Commons Attribution License, which permits unrestricted use, distribution, and reproduction in any medium, provided the original work is properly cited.

Several microstructures are evolved at the interface when sparingly soluble solid surfactants come in contact with water. One class of these microstructures is termed as "myelin figures"; these were observed when phosphatidylcholine came in contact with water. Although the myelins are initially simple rod-like, complex forms like helices, coils and so forth. appear in the later stage. Finally, the myelins fuse together to form a complex mosaic-like structure. When studied by taking a cross-section using cryoscanning electron microscopy, it revealed concentric circular pattern inside the myelin figures. The cross-sections of (dioctyl sodium dodecyl sulfosiccinate) AOT/water system myelin internal structures were lost. When p-toluenesulfonic acid (PTS) $2 \mathrm{wt} \% \mathrm{was}$ present in the water phase, AOT myelins revealed the internal microstructures. It has annular concentric ring-like structure with a core axon at the centre. Further investigation revealed new microstructures for the first time having multiple axons in the single-myelin strand.

\section{Introduction}

Myelin figures are formed when some surfactants come in contact with water. These synthetic structures resemble the myelin structures in nerve systems. Unfortunately, these are transient nonequilibrium structures that sometimes are even difficult to reveal easily. Depending on the ability of surfactants to form oriented monolayers, micelles, vesicles, and a versatile phase behavior and diversity in colloidal structures, surfactants find utility in almost each and every industry, directly or indirectly as a basic component of most cleansing agents such as detergents. Besides, they are one of the key components of many biological systems. Such vast applications necessitate the study and understanding of the observed dynamic and equilibrium aggregates or the microstructures of surfactants in solutions for better commercial applications. At higher concentrations the amphiphiles can become spontaneously organized into mesophases (or lyotropic liquid crystal phases). The most common of these are the hexagonal phase, in which amphiphiles assemble into long cylinders arranged in a hexagonal pattern, and the lamellar phase, in which amphiphiles assemble into bilayers that stack parallel to each other. Surfactants are primarily used in aqueous solutions and are often present in lamellar phase in the initial formulation or arise spontaneously on the first contact. Enhancing the performance of such products relies on a scientific understanding of the dissolution behavior. During the dissolution in solvent, some surfactants display various types of non-equilibrium microstructures or instabilities at the interface as they progress towards an equilibrium state. One such interesting and fascinating non-equilibrium microstructure is the classical "myelin figure," first observed in lipids by Virchow in 1854 [1, 2]. L. N. Zou [3] suggests that myelin formation is due to mechanical instability, since the mechanical properties of the lamellar structure are 
anisotropic (depending on the direction, it behaves either as a fluid or as an elastic solid), the myelin instability is one that has both fluid and elastic characteristics. The fluid flow of surfactant, driven by the hydration gradient, results in the formation and growth of myelins [3]. Myelin figure formation is important as a model for understanding the self-assembly and self-organization in biological membranes. Myelin figures of the egg-yolk phosphatidylcholine/water system were oriented in a magnetic field due to the diamagnetic anisotropy of the molecules by bending at the roots with the long axes parallel to the field. It might have some effect in an actual biological system with sufficient ordering and size. The actual tissue has far more complicated components than the myelin figure and is usually connected with surrounding tissues. Since, an orienting effect on the tissue, though small, can be expected [4]. In further studies, Mishima and Morimoto have reported that myelin figures orient themselves on application of an alternating electric field [5]. Their behavior in the alternating field is very similar to that of the biological cells. Experiments were also performed to study the effect of additives (silica) to lamellar phase on diffusion coefficients $[6,7]$. It has been observed that the presence of silica particles causes extensive coiling of myelin structures. Due to the strong concentration gradients, the interface between pure surfactant and pure water can consist of multiple domains of different phases, as well as metastable structures whose origins are not understood, evolving in spatially and temporally complex ways [8-11]. Huang et al. showed that even a slight increase in the overall hydration can overcome the added elastic energy of forming a myelin. In this sense, the hydration gradient also plays a role in myelin formation analogous to the force of gravity in pendant drop breakup [12]. The swelling and dissolution dynamics of the myelin growth in the surfactants Aerosol-OT (AOT) and phosphatidylcholine (PC) in aqueous medium have been studied $[13,14]$. The effect of the additive $p$ toluenesulfonic acid (PTS) on the dynamics and the shape of these myelin structures has been investigated. This molecule was chosen, since PTS acts as a bilayer penetrating agent. They observed not only the effect on growth but also its effect on the coiling of myelins. In earlier stage a large number of looped rods were observed which at later stages formed single and double helices, tadpoles, and so forth. Single helices observed had pitches almost equal to the rod diameter. Frette et al. [15] have used hydrophilic dextran polymer as the bilayer anchoring agent to study the coiling instability in PC and have concluded that the polymer added changes the spontaneous curvature in the membrane. They have, using a model, argued that coiling instabilities are formed beyond a critical concentration. Further, Chen and Tsujii [16] have successfully immobilized these myelin figures in poly(acrylamide) gel and are stable for at least 6 months. These myelin figures provide useful information as models to study myelins as nerve systems. Later, Lin et al. [17] studied the effect of monovalent electrolytes on the myelins formed with negatively charged lipids. They are not sensitive to the specific ion types of added electrolytes.

Myelins are elongated multilamellar rod-like structures in which bilayered lamellae of amphiphiles are coaxially stacked around the rod-axis with liquid medium in between. These are observed in MBP (myelin basic protein) when a highly immiscible lamellar phase (of lipids or surfactants) is contacted with the excess of aqueous medium. Myelin figures are highly viscous, gel-like microstructures whose structural features are essentially similar to that of the nerve myelin sheath, a white material insulating nerves.

The phenomena of formation of the myelin structure is useful for understanding many of the biological processes which take place in animals and humans. The important biological processes they associate with can gauge the importance of these non-equilibrium microstructures. Myelin sheaths insulate the axon membrane of the nerves and prevent the leakage of electrical impulses into the surrounding fluid as well as provide the structural support for the nerve axon. When myelin sheath is damaged or destroyed by disease, nerve impulses are slow and inefficient. During the critical period of neurological damage, the myelin formation is high compared to that without injury. Also under exposure to radiation, the levels of the myelin associated with proteins like MBP were found to decrease soon after the radiation dosage.

Owing to their wide involvement in the biological processes, much literature is available on the biological aspects of myelin. The myelin growth is also observed in the surfactant-solvent system. A scientific study of their interaction with solvent surrounding during dissolution process is necessitated to enhance the performance of these surfactant products. But yet their evolutionary process and shape fluctuations with change in surrounding environment and time remain largely unexplored. Most of the literature has been confined to the study of diffusion aspects of their growth, like Sakurai and Kawamura [18] which have established the myelin growth as an aggregate diffusion growth in the initial stages of surfactant-solvent contact. But these models have been unable to account for the instabilities and fluctuations like coiling of myelin. Sakurai et al. [1921] have observed the cross-sections of myelin figures in egg-yolk phosphatidylcholine/water system directly by using cryo-SEM. The present work is a new systematic study of myelin figure microstructures, Aerosol-OT in presence of bilayer penetrating agent (PTS) and in continuation of soybean PC similar to Sakurai et. al. on myelin figures of PC extracted from egg-yolk [19]. PC myelins readily revealed internal microstructures in the presence of water, but AOT microstructures are delicate and need great care to keep intact myelin tubular microstructures in water alone. We were unable to visualize internal microstructures after taking vertical cross-section of myelins. Recent structural confirmation studies of myelin figures [20] revealed that denser parent stack on hydrophobic substrate at low temperature can produce more stable myelin figure with greater life time. Bilayer penetrating agent (PTS) solution in water was used for the first time to elucidate the internal microstructure of Aerosol-OT by controlling the viscoelastic properties, which was not possible by water alone. Another new finding we want to report is the presence of multiple axons of bilayers in a single myelin figure. These interesting new microstructures will help us for potential applications in material science such 
as surfactant preparation of template nonporous and porous composite materials, which will be utilized in catalytic processes and gas occlusion. AOT can be used in printing ink due to rapid and dramatic lowering in surface tension of the formulation, leading to improved wetting of the substrate. This, in turn, improves adhesion, gloss, and color resolution. Rapid wetting can be compatible with very fast printing rates. The internal non-equilibrium microstructures are exhibited due to important property of surfactant systems, that is, dynamic surface tension. Surfactant molecules first diffuse from the bulk to the surface, then adsorb. Therefore, a freshly formed interface of a surfactant solution has a surface tension very close to that of the solvent, and this dynamic surface tension will then decay over a certain period of time. Therefore, these initial dynamic microstructures were investigated by cryoscanning electron microscopy.

\section{Materials and Methods}

All pure-grade chemicals were used as procured. Phosphatidylcholine and AOT from Sigma Chemical Co. (St. Louis, MO, USA) were used without further purification. Chloroform (purity 99.5\%) from Sisco Research Lab. Pvt. Ltd., India, is used as casting solvent, bilayer penetrating agent which is p-toluene-sulphonic acid from Sisco Research Lab. Pvt. Ltd., India, liquid nitrogen, Freon R-22, gold wire, and camera roll 120 was from Rollei Agfa Universal 200 film. This study of microstructures was carried out by optical, video-enhanced microscopy and cryo-scanning electron microscopy to view the internal microstructures in Aerosol-OT and phosphatidylcholine.

2.1. Cryoscanning Electron Microscopy. The scanning electron microscopy micrographs were used to study the surface topography of the non-equilibrium myelin structures that were formed. All experiments were carried out using the JEOL, Japan, model JSM-6400 scanning electron microscope with cryogenic attachments enabling sample preparation in liquid nitrogen [22]. Cryoduers were filled with liquid nitrogen and allowed to cool both, the main stage and cryoairlock chamber stage to $-150^{\circ} \mathrm{C}$. Sample mounting troughs were initially filled with liquid nitrogen. For preparing the sample, 2-3 water/solution drops were added to the lump of surfactant stuffed in the half-holed stub and myelin figures were allowed to grow for 2 minutes. Meanwhile, Freon 22 was poured into another container. Myelin growth was then vitrified by rapidly plunging into a pool of liquid Freon 22 using spring-loaded plunging device [23]. After $5 \mathrm{~min}$ of temperature stabilization, the sample stub was transferred to the liquid nitrogen trough. Here the sample holder was mounted in the trough. The sample-inserting rod was screwed to the sample holder and inserted quickly into precooled airlock chamber stage. It was placed on a previously cooled main stage at $-150^{\circ} \mathrm{C}$ of the cryo-SEM and was allowed to stabilize to the internal environment for about 20-25 minutes. The frost formed on sample evaporates, and the sample surface is clearly visible. We can also remove the frost by using defrost switch that heats the main sample stage. But, during this process one has to be careful and do not allow the temperature to go above $-110^{\circ} \mathrm{C}$ or it will then initialize the crystallization process, which will damage the internal microstructures of our interest. When frost is evaporated from the sample surface, it is then cryofractured using an internal knife and further coat the sample with gold evaporator attached on the cryounit inside the microscope. Sample was, thus, ready to view the cross-section under SEI mode. The micrographs were further analyzed using freeware image processing software gimp 2.6.

\section{Results and Discussion}

On the basis of our preliminary experiments, soybean phosphatidylcholine and Aerosol-OT were selected as surfactants for experimenting, since both show non-equilibrium myelin structures when they come in contact with water. This type of dissolution by myelin formation is observed mostly in less readily water soluble surfactants. Other surfactant molecules like $\mathrm{CTAB}$ and SDS do not dissolve forming myelin figures.

\subsection{Myelin Growth in Phosphatidylcholine (PC). Sakurai and} Kawamura [18] have studied the morphology and growth behavior of myelin figures in egg-yolk phosphatidylcholine or lecithin. They observed that as soon as the water comes in contact with lecithin, myelin figures start growing. According to them, myelin growth is a three-step process based on the time of growth. In the first step, myelin figures involve the diffusion of an aggregate of lecithin molecules that are simple rod-like and grow all together perpendicularly to the lecithin-water surface. The second step of growth process takes place by folding and coiling by lateral diffusion in the bilayer membranes. When water was contacted to solid PC, myelin figures start growing spontaneously from the surfactant/water interface. Initially myelins were simple rod-like and come out almost perpendicular to the surfactant/water interface. Growth behaviors of myelin figures in this paper are from soybean phosphatidylcholine (a natural surfactant). Micrograph (Figure 1(a)) shows the rod shaped myelin freeze dried without taking cross-section using the cold knife, but coated the sample with gold by evaporator for viewing microstructure growing in the direction indicated with an arrow. Tip region of the myelin is club-shaped with an average diameter of approx. $21 \mu \mathrm{m}$ and rare part is rod-like with an average diameter of approx. $7.70 \mu \mathrm{m}$. The next stage with "double-helix" spirally coiled myelin figures is shown by an arrow (Figure 1(b)). The external surface morphology in myelin figures is porous in some areas. In this stage of coiling the average diameter is slightly more approx. $8.9 \mu \mathrm{m}$. In continuation, these PC myelin cross-sections were taken and the internal microstructures (Figure 2) were observed. Similar cross-sections were observed by Sakurai et al. [19] in egg-yolk phosphatidylcholine/water system. In order to obtain more detailed micrographs of the cross-sections, the water molecules, which existed in the water cores as well as in the water layers trapped in the stacked bilayers and in the gap between neighboring myelin were evaporated at $-110^{\circ} \mathrm{C}$ for $10 \mathrm{~min}$. Both the micrographs (Figures 2(a) and 2(b)) 

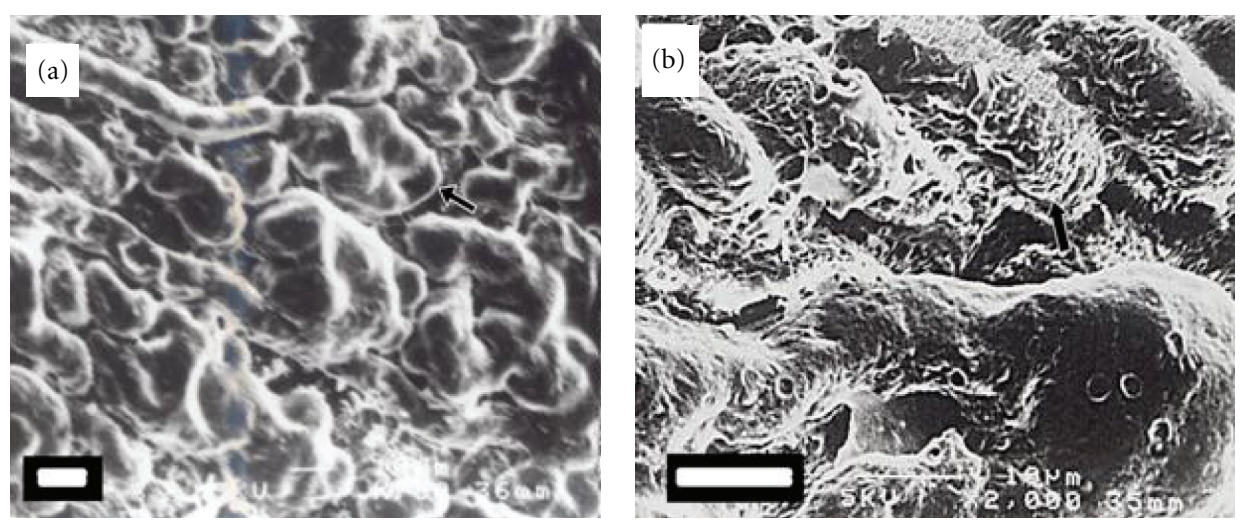

FIGURE 1: Micrographs show outer surface of PC myelin with accelerating voltage of $5 \mathrm{kV}$. The surface of specimen was treated by a vacuum deposition of gold after etching. (a) PC myelin figure growing in the direction of arrow. (b) The "double-helix" coiling of PC myelin figure consists of two simple myelin rods having the same diameter indicated by an arrow. The white bar in the micrograph represents $10 \mu \mathrm{m}$.
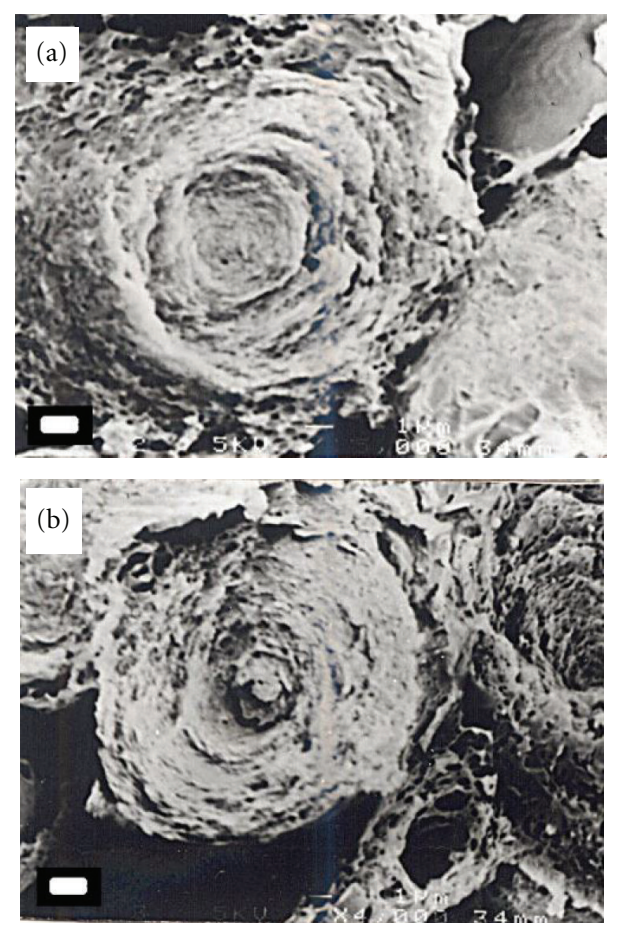

FIGURE 2: A cross-sectional view of the growing tip region in PC myelin growth with water after freeze drying and plunging in liquid nitrogen. Both micrographs (a) and (b) show concentric lipid bilayers with a single axon, accelerating voltage of $5 \mathrm{kV}$ was used. The white bar in the micrograph represents $10 \mu \mathrm{m}$.

show concentric arrangement of lipid bilayers with a single central axon clearly visible. The diameter of these myelin cross-sections is approx. $12 \mu \mathrm{m}$. The water cores at axon of myelin are slightly off-centered. It can be interpreted from the cross-sections that the lateral diffusion type of process takes place within the PC bilayers to assist the molecular movement towards the tip of the myelin figures.

3.2. Myelin Growth in Aerosol-OT with Water. In case of phosphatidylcholine extract from soybean, the myelin can be easily seen than that of Aerosol-OT (an artificial surfactant). Similar to our earlier experiment carried out in PC, we also freeze myelin growth in AOT with water as seen in Figure 3. Myelin figures formed in AOT are delicate and fragile as the walls are ruptured. Great care has to be taken while plunging the AOT myelin. Yet, we managed to take the crosssection of AOT myelin figures. Lateral view of the bundled myelin figures (Figure 3(a)) can be observed along with the top cross-sections of myelin. They had an average diameter of approx. $10-15 \mu \mathrm{m}$. A cross-sectional view (Figure 3(b)) was magnified to observe the internal microstructures shown by an arrow. Unfortunately, the internal microstructures are ruptured and the details are lost. The outer layer is peeled off sometimes, perhaps due to plunging in Freon 22 and later immersed in liquid nitrogen. It may also be that the internal structures are lost as cross-section was cut with the cold knife. There is no previous work done to directly visualize frozen internal microstructures of AOT myelins by cryoSEM. We were unable to obtain internal microstructures of $\mathrm{AOT}$ in water alone. The necessity of a stabilizing additive is evident to view the internal detailed microstructures. So, further experiments were carried out using $\mathrm{p}$-toluenesulfonic acid, a bilayer penetrating agent.

3.3. Myelin Growth in Aerosol-OT with PTS ( $2 w t \%) /$ Water. p-toluenesulfonic acid (Figure 4), which is a bilayer penetrating agent is also a hydrotropic, should be ideally suitable for modifying rigidity. It was useful to know the role of rigidity in the lamellar membranes of myelin formation. Previous studies have revealed that in the presence of PTS, myelin growth rate is affected as anticipated. There was a drastic change in the nature of myelin formation by the spectacular formation of coiling instabilities. However, using PTS concentration above $2 \mathrm{wt} \%$, a large number of complex structures like coils and helices start forming. Initially a large number of looped rods were observed which at later stage formed single and double helices, tadpoles, and so forth. Single helices observed had pitches almost equal to the rod diameter. Acid addition salts are prepared from an active agent in the free-base form (e.g., compounds having 

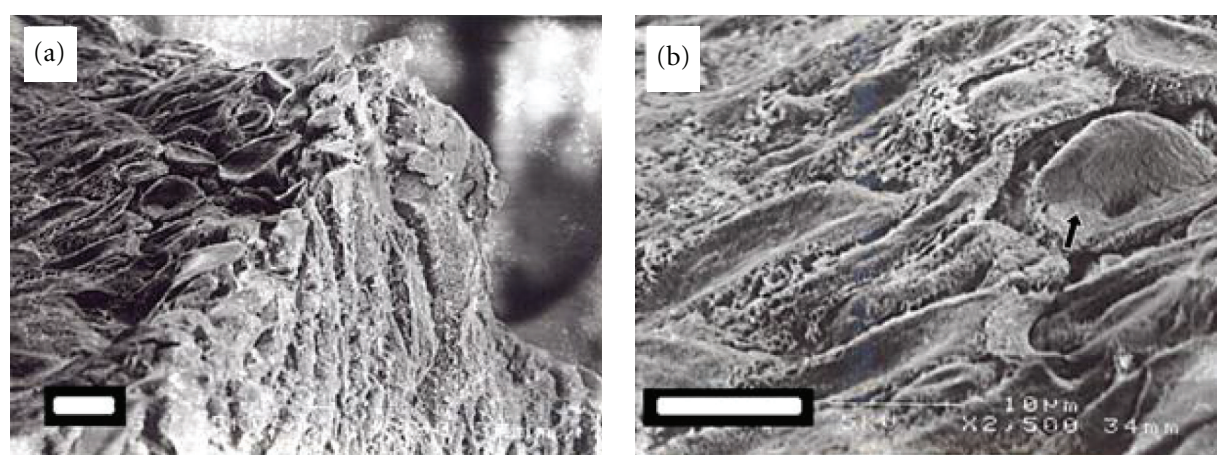

FIGURE 3: Micrographs with cross-sections of AOT myelin growth in water, later freeze-dried after plunging in liquid nitrogen and observed under SEM. Surface of the specimen was treated by a vacuum deposition of gold. (a) Lateral view of elongated tubular AOT myelin figures bundled with tip region after cutting with a cold knife. (b) A cross-sectional view of AOT myelin figures showing no detailed internal microstructures indicated by arrow. The white bar in the micrographs represents $10 \mu \mathrm{m}$.

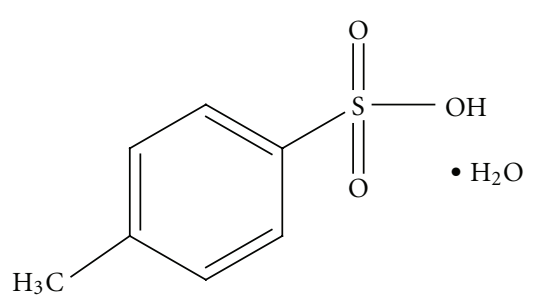

Figure 4: Molecular structure of p-toluenesulfonic acid, which is a bilayer penetrating agent.

a neutral $-\mathrm{NH}_{2}$ group) using conventional means, involving reaction with a suitable acid. Suitable acids for preparing acid addition salts include organic acids, for example, acetic acid, propionic acid, p-toluenesulfonic acid, and salicylic acid. An acid addition salt may be reconverted to the free base by treatment with a suitable base. It appears that the formation of new instabilities require a threshold concentration of about $2 \mathrm{wt} \%$ PTS. In the present study, we have taken the PTS in the water phase. It has helped us to reveal the internal microstructures of myelin figures, and newer structures were observed for the first time in case of AOT myelins. As seen in Figure 3, the Aerosol-OT solutions in water alone were unable to reveal internal microstructures. Previous studies in addition of silica resulted in an extensive increase in the number of helical structures compared with that in a pure surfactant-water system and to the surfactant phase reduces the diffusion coefficient [13, 14]. Another additive used was the bilayer penetrating agent (PTS) that gave some interesting results. Firstly, the growth rate was affected resulting in coiling initially and in the later stages formed single and double helices, tadpoles, and so forth [7]. Instead of water, solution with paratoluene sulphonic acid was added. Rest of the procedure was followed as discussed earlier. The optimum concentration of PTS was $2 \mathrm{wt} \%$ in Figure 5 that gave us the better understanding and view of internal microstructures.

A cross-sectional view of many myelin growth structures (Figure 5(a)) was observed with detailed internal microstructures. It is evident that PTS plays a crucial role for the rigidity of lamellar membranes in myelin formation. In another micrograph (Figure 5(b)), annular concentric ringlike microstructures with a single core axis at the center are observed. In further studies, we were able to visualize for the first time these new microstructures, in which single myelin figures having multiple core axons like microstructures. Earlier literature shows that cross-section of myelins in PC are single concentric ring-like structures that were obtained by Sakurai et al. [19]. Some peculiar fusion and connection was observed among the stacked bilayers between three different myelin rods. Polarizing optical microscope could not reveal the connectivity between the fused inner regions of the myelin figures. These stacked bilayers inner regions were connected with one another and found mixing bilayers [21].

In contrast to these we here find that in case of AOT in presence of PTS/solution there were some multiple axons in single-myelin figure along with single-axon myelins. Schematic representation in Figure 6 show the single Aerosol-OT myelin figure containing three axons having ring-like bilayered microstructures each. There were also some bilayers mixing observed in between the two axons. The amplified view of the bilayer shows the arrangement of the internal lipids in water/PTS solution. Each myelin figure consists of a cylindrical role of stacked bilayers and water was embedded in between them, which are alternately wrapped existing from the outer surface of myelin figures to the center core. In continuing the cross-sectional view studies (Figure 7(a)), we were able to visualize these microstructures in single-myelin strand containing multiple-core axons. This is a new phenomenon observed. There are two myelin figures seen one with two axons and the other with three axons indicated by arrows. The internal microstructure (Figure 7(b)) details were visualized by magnifying singlemyelin figure. The AOT myelin figure could be visualized with greater details and a greater contrast revealing the three cylindrical bilayers with three axons at the center core indicated by arrows. The average diameter of entire myelin figure is approx. $33.0 \mu \mathrm{m}$ which is very well in the range of a single myelin strand and the individual core lipid bilayers diameter are ranging from 12 to $15 \mu \mathrm{m}$. We suppose that the cryo-SEM technique utilized here is helpful in clarifying the 

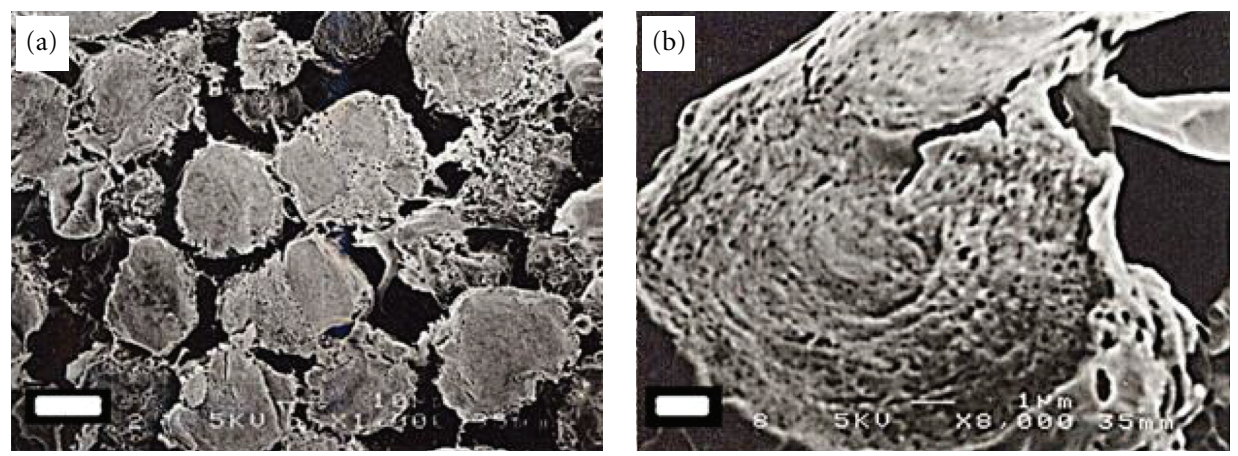

FIGURE 5: A cross-sectional view of AOT myelin growth in PTS/water solution (2 wt \%) freeze-dried after plunging in liquid nitrogen. (a) A cross-sectional view of several AOT myelin figures with detailed internal microstructures visible. (b) A cross-sectional view of single-myelin figure with concentric lipid bilayers similar to PC myelin figure. The white bar in the micrograph represents (a) $10 \mu \mathrm{m}$ and (b) $1 \mu \mathrm{m}$.

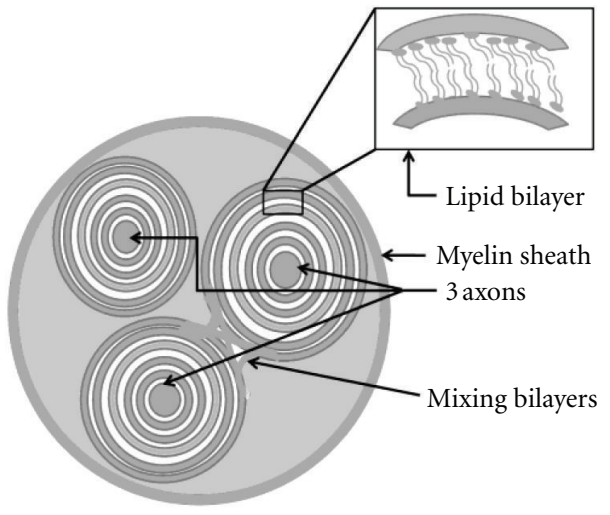

FIgUre 6: Schematic diagram of AOT/PTS single myelin figure containing three axons and mixing of bilayers. Zoom in portion shows the amplified view of lipid bilayer.

new internal microstructures of complicated myelin figures, which will further help us to understand the mechanism of spontaneously organized mesophases and self-assembly of lipids into cell membranes.

To understand the phenomenon, one must understand how micellization occurs. In the micellization process, molecular geometry plays an important role, and it is essential to understand how surfactants can pack. The main structures encountered are spherical micelles, vesicles, bilayers, or inverted micelles. Two opposing forces control the self association process: hydrocarbon-water interactions that favour aggregation (i.e., pulling surfactant molecules out of the aqueous environment) and head group interactions that work in the opposite sense. These two contributions can be considered as an attractive interfacial tension term due to hydrocarbon tails and a repulsion term depending on the nature of hydrophilic group.

Further quantification done by Mitchell and Ninham [24] and Israelachvili [25] concludes that aggregation of surfactants is controlled by a balanced geometry. The overall free energy of association depends on three critical geometric terms given by

$$
\frac{v}{\left(a^{\circ} R_{\text {mic }}\right)}=\frac{1}{3}
$$

where $v$ is the volume of hydrophilic tail(s), $a^{\circ}$ is the minimum interfacial area occupied by the head group, and $R_{\text {mic }}$ is micelle core radius

$$
\frac{v}{\left(a^{\circ} \cdot I_{c}\right)} \leq \frac{1}{3}
$$

where $I_{c}$ is the maximum extended chain length of the tail in the micelle core. This defines a critical packing parameter, $P_{c}$, as the ratio of volume to surface area:

$$
P_{c}=\frac{v}{\left(a^{\circ} \cdot I_{c}\right)}
$$

The parameter $v$ varies with the number of hydrophobic groups, chain unsaturation, chain branching, and chain penetration by other compatible hydrophobic groups, while $a^{\circ}$ is mainly by electrostatic interactions and head group hydration. $P_{c}$ is a useful quantity, since it allows the prediction of aggregate shape and size. For $P_{c}$, between 0.33 and 0.5 , large cylindrical or rod-shaped micelles are obtained. For double-chain surfactants like AOT, PC ranges from 0.5 to 1.0 , where flexible bilayers structures can be obtained. When water interacts with surfactant, hydration forces act on it and with increase in aggregation micelles get closer to one another. To maximize separation, the shape and size of micelles change and mesophases or lyotropic structures are formed.

\section{Conclusion}

New internal microstructures were revealed by allowing bilayer penetrating agent to come in contact with AOT. In the present work, we reveal new and interesting multiple axons in the single myelin figure of AOT. Internal microstructures of soybean PC showing rod-like and complex-form helices 

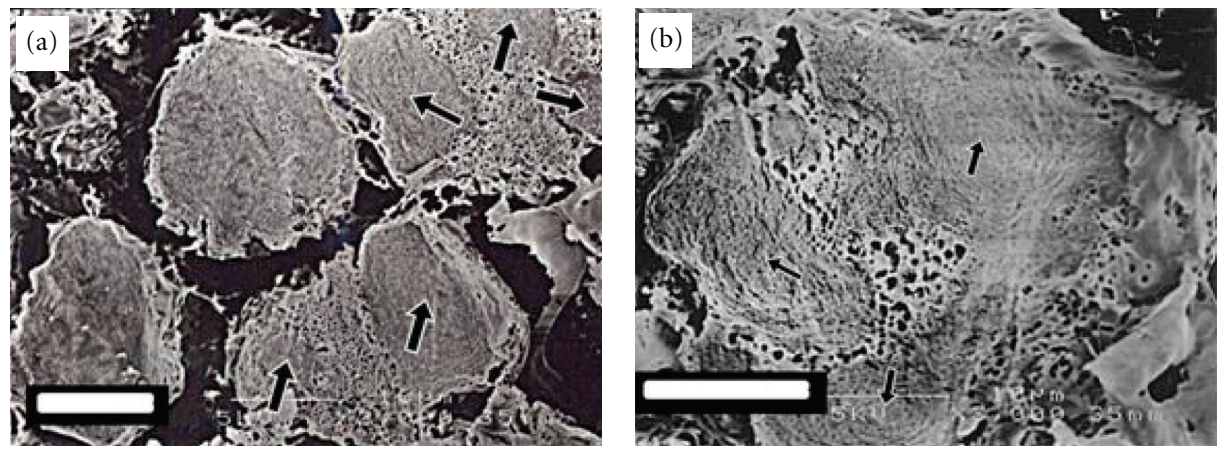

FIGURE 7: A cross-sectional view of the growing AOT myelin figure in the presence of $2 \mathrm{wt} \%$ p-toluenesulfonic acid solution as bilayer penetrating agent, accelerating voltage of $5 \mathrm{kV}$ was used. The surface of the specimen was treated by a vacuum deposition of gold. (a) A cross-section of several AOT myelin figures with single- and multiple-core axons shown by arrows are observed. (b) The internal structures of AOT single-myelin figure revealing the three concentric lipid bilayers with three axons indicated by arrows. The white bar in the micrograph represents $10 \mu \mathrm{m}$.

like coiling that appear in the later stages were easily obtained, cross-sectional view of PC/water myelin also shows concentric arrangements of lipid bilayers. In case of AOT (synthetic surfactant), internal microstructures were fragile and lost with water alone. They were revealed for the first time with a bilayer penetrating agent (PTS) solution in water. This phenomenon of myelin formation is observed mostly in more hydrophobic surfactants. PTS plays a key role in controlling the viscoelastic properties, and bilayer rigidity is important in myelin growth of AOT. Cross-sectional views of AOT myelins also show clearly 2-3 multiple axons in some single-myelin figures possessing concentric bilayers.

\section{Acknowledgment}

J. Bellare and M. K. Temgire gratefully acknowledge financial support from the Unilever Industries Pvt. Ltd.

\section{References}

[1] R. Virchow, "Ueber das ausgebreitete Vorkommen einer dem Nervenmark analogen Substanz in den thierischen Geweben," Archiv für Pathologische Anatomie und Physiologie und für Klinische Medicin, vol. 6, no. 4, pp. 562-572, 1854.

[2] H. Kelkar, "History of liquidcrystals," Molecular Crystals and Liquid Crystals, vol. 21, pp. 1-48, 1973.

[3] L. N. Zou, "Myelin figures: the buckling and flow of wet soap," Physical Review E, vol. 79, no. 6, Article ID 061502, 10 pages, 2009.

[4] I. Sakurai and Y. Kawamura, "Magnetic-field-induced orientation and bending of the myelin figures of phosphatidylcholine," Biochimica et Biophysica Acta, vol. 735, no. 1, pp. 189-192, 1983.

[5] K. Mishima and T. Morimoto, "Electric field-induced orientation of myelin figures of phosphatidylcholine," Biochimica et Biophysica Acta, vol. 985, no. 3, pp. 351-354, 1989.

[6] A. Chowdhury, Optical microscopy and scanning electron microscopy of surfactant myelins [M.S. thesis], 2001.

[7] M. Haran, A. Chowdhury, C. Manohar, and J. Bellare, "Myelin growth and coiling," Colloids and Surfaces A, vol. 205, no. 1-2, pp. 21-30, 2002.
[8] M. Buchanan, J. Arrault, and M. E. Cates, "Swelling and dissolution of lamellar phases: role of bilayer organization," Langmuir, vol. 14, no. 26, pp. 7371-7377, 1998.

[9] M. Buchanan, S. U. Egelhaaf, and M. E. Cates, "Dynamics of interface instabilities in nonionic lamellar phases," Langmuir, vol. 16, no. 8, pp. 3718-3726, 2000.

[10] M. Buchanan, L. Starrs, S. U. Egelhaaf, and M. E. Cates, "Kinetic pathways of multiphase surfactant systems," Physical Review E, vol. 62, no. 5, pp. 6895-6905, 2000.

[11] M. Buchanan, S. U. Egelhaaf, and M. E. Cates, "Swelling and dissolution of onion phases: the effect of temperature," Colloids and Surfaces A, vol. 183-185, pp. 293-302, 2001.

[12] J. R. Huang, L. N. Zou, and T. A. Witten, "Confined multilamellae prefer cylindrical morphology-a theory of myelin formation," European Physical Journal E, vol. 18, no. 3, pp. 279-285, 2005.

[13] H. Dave, M. Surve, C. Manohar, and J. Bellare, "Myelin growth and initial dynamics," Journal of Colloid and Interface Science, vol. 264, no. 1, pp. 76-81, 2003.

[14] G. Hota, J. R. Bellare, K. C. Khilar, and C. Manohar, "Reactive organization of particles into macroscopic length scales by myelin growth," Current Science, vol. 80, no. 11, pp. 14061407, 2001.

[15] V. Frette, I. Tsafrir, M. A. Guedeau-Boudeville, L. Jullien, D. Kandel, and J. Stavans, "Coiling of cylindrical membrane stacks with anchored polymers," Physical Review Letters, vol. 83, no. 12, pp. 2465-2468, 1999.

[16] X. Chen and K. Tsujii, "Synthetic myelin figures immobilized in polymer gels," Soft Matter, vol. 3, no. 7, pp. 852-856, 2007.

[17] M. Lin, L. Li, F. Qiu, and Y. Yang, "Effect of added monovalent electrolytes on the myelin formation from charged lipids," Journal of Colloid and Interface Science, vol. 348, no. 2, pp. 505510, 2010.

[18] I. Sakurai and Y. Kawamura, "Growth mechanism of myelin figures of phosphatidylcholine," Biochimica et Biophysica Acta, vol. 777, no. 2, pp. 347-351, 1984.

[19] I. Sakurai, T. Suzuki, and S. Sakurai, "Cross sectional view of myelin figures," Biochimica et Biophysica Acta, vol. 985, pp. 101-105, 1989.

[20] L. Tayeba, M. Mozafari, D. Vashaee, and A. N. Parikh, "Structural configuration of myelin figures using fluorescence microscopy," International Journal of Photoenergy, vol. 2012, Article ID 685617, 7 pages, 2012. 
[21] I. Sakurai, T. Suzuki, and S. Sakurai, "Structure and growth behaviour of myelin figures," Molecular Crystals and Liquid Crystals B, vol. 180, pp. 305-311, 1990.

[22] E. M. Slayter and H. S. Slayter, Light and Electron Microscopy, Cambridge University Press, New York, NY, USA, 1992.

[23] K. Bhadriraju and J. Bellare, "A cryo-SEM technique developed and applied to surfactant liposomes," in Proceedings of the 51st Annual Meeting of the Electron Microscope Society of America, pp. 284-285, August 1993.

[24] D. J. Mitchell and B. W. Ninham, "Micelles, vesicles and microemulsions," Journal of the Chemical Society, Faraday Transactions 2, vol. 77, no. 4, pp. 601-629, 1981.

[25] J. N. Israelachvili, Intermolecular and Surface Forces, Academic Press, London, UK, 1985. 


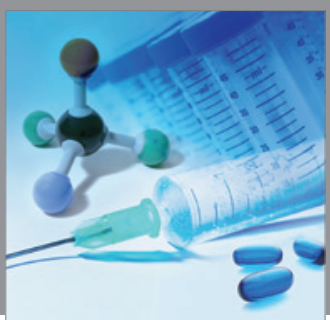

International Journal of

Medicinal Chemistry

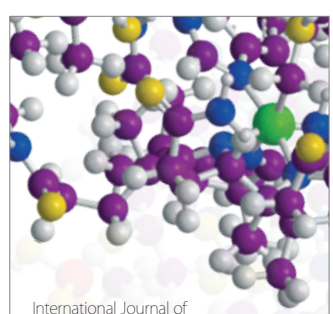

Carbohydrate Chemistry

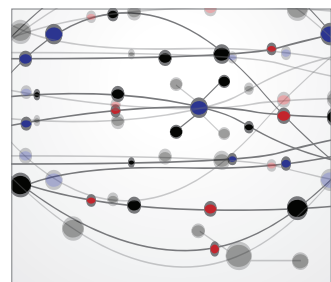

The Scientific World Journal
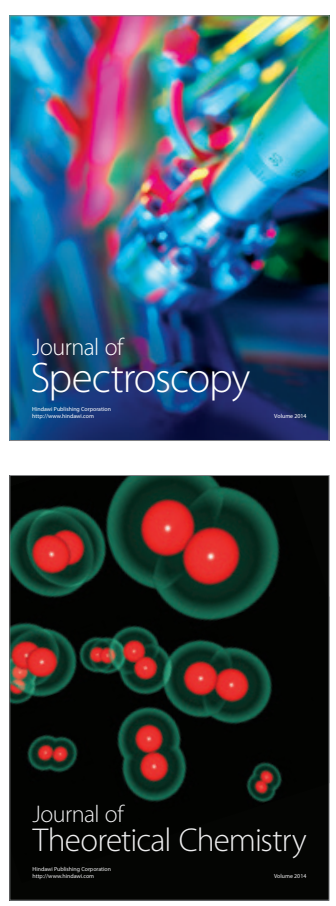
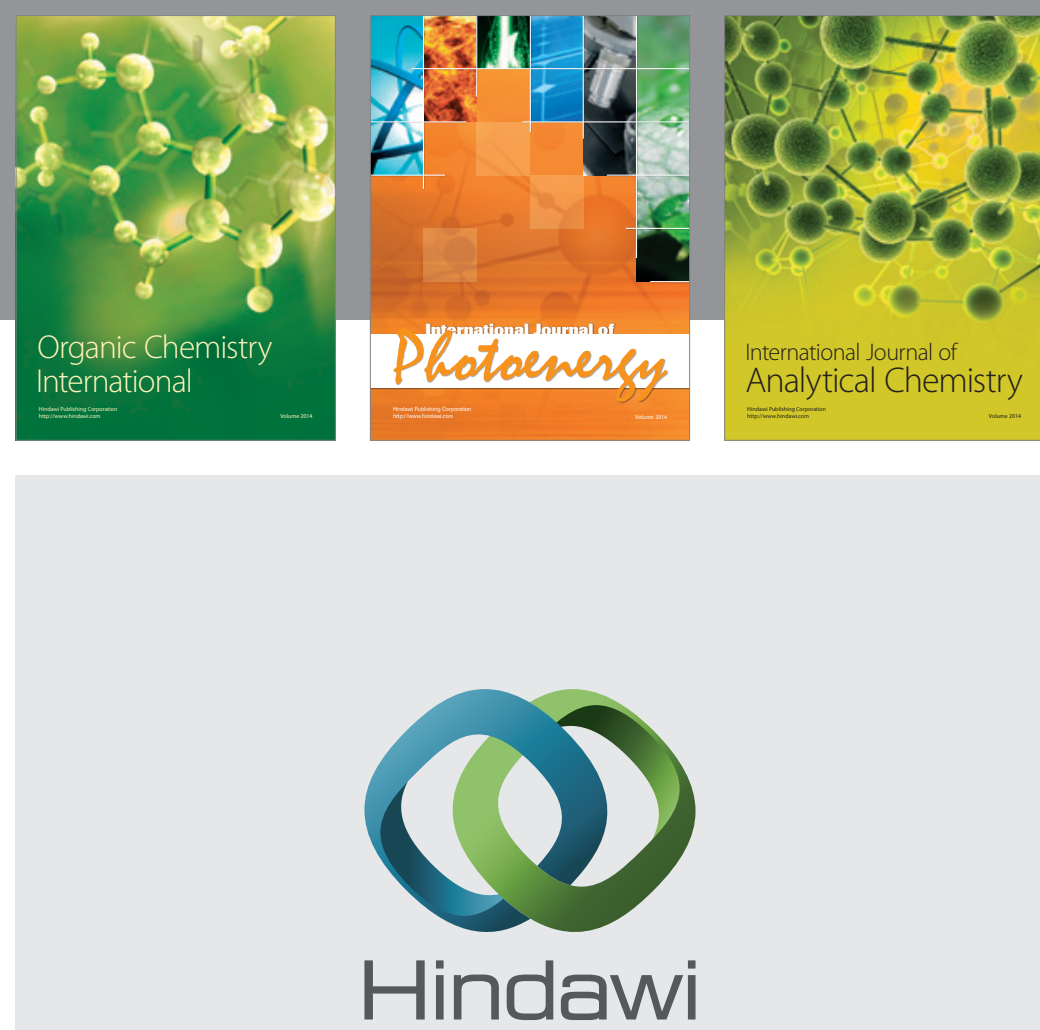

Submit your manuscripts at

http://www.hindawi.com
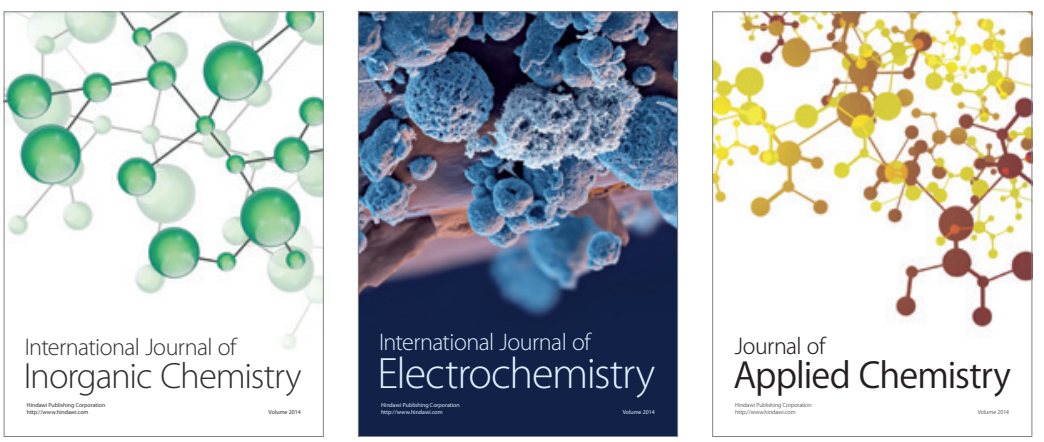

Journal of

Applied Chemistry
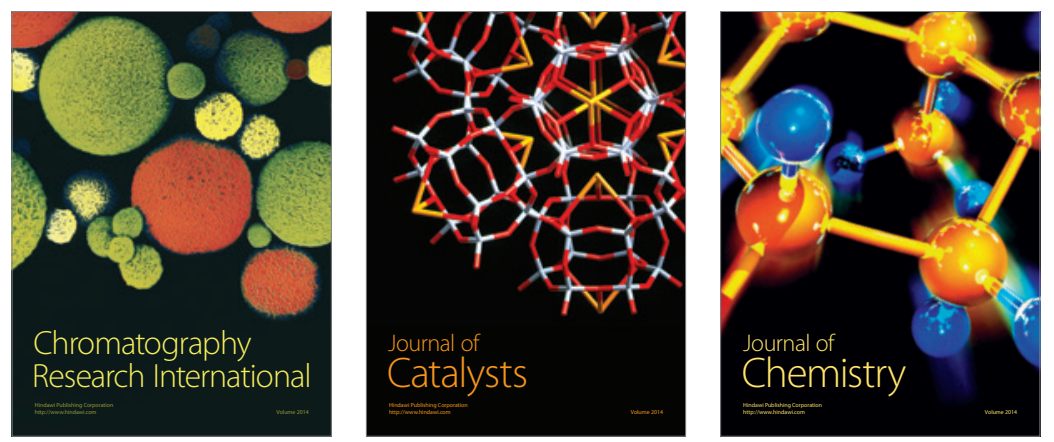
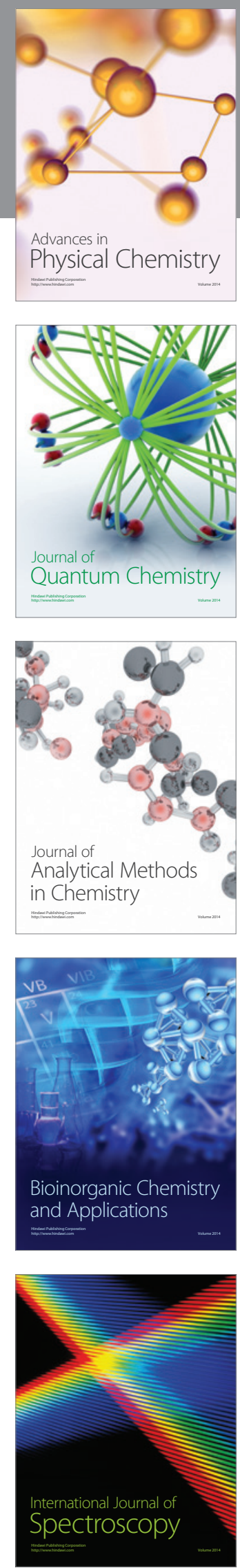